Wien klin Mag 2016 · 19:154-169

DOI 10.1007/s00740-016-0139-9

Online publiziert: 16. September 2016

@ ( Der/die Autor(en) 2016. Dieser Artikel ist eine Open-Access-Publikation.

CrossMark

\author{
W. Fartek' · G. Gemes ${ }^{2} \cdot$ M. Hiesmayr ${ }^{3}$ B. Kabon ${ }^{3}$. S. Kozek-Langenecker ${ }^{4}$. \\ R. Likar ${ }^{5} \cdot$ G. Luckner ${ }^{6} \cdot$ W. Mitterndorfer ${ }^{7} \cdot$ C. Torgersen ${ }^{8}$ \\ ${ }^{1}$ LKH Hartberg, Hartberg, Österreich \\ ${ }^{2}$ LKH-Universitätsklinikum Graz, Graz, Österreich \\ ${ }^{3}$ Medizinische Universität Wien, Wien, Österreich \\ ${ }^{4}$ Evangelisches Krankenhaus Wien, Wien, Österreich \\ ${ }^{5}$ Klinikum Klagenfurt, Klagenfurt, Österreich \\ ${ }^{6}$ Universitätsklinik Innsbruck, Innsbruck, Österreich \\ ${ }^{7}$ Kepler Universitätsklinikum Linz, Linz, Österreich \\ ${ }^{8}$ LKH Salzburg, Salzburg, Österreich
}

\title{
Stellenwert von Hydroxyethylstärkelösung in der Volumenersatztherapie
}

\section{Ergebnisse eines österreichischen Expertentreffens}

\section{Teil 1: Status quo der intravasalen Volumentherapie}

\section{Einleitung}

Die intravasale Volumentherapie ist eine der häufigsten therapeutischen Maßnahmen im periinterventionellen Setting. Sie wird insbesondere bei Patienten mit akuter Hypovolämie bzw. im Schock oder bei hämodynamischer Instabilität durchgeführt, um ein ausreichendes intravasales Volumen und Sauerstoffangebot sicherzustellen und konsekutiv eine adäquate Pumpleistung des Herzens, Gewebeoxygenierung, Gewebeperfusion und Organfunktion zu erreichen [1].

Zur Behandlung einer intravasalen Hypovolämie werden kristalloide und kolloidale Infusionslösungen eingesetzt. Kolloide sind seit der Erstanwendung von Gelatine zu Beginn des 20. Jahrhunderts ein fester Bestandteil im perioperativen Bereich sowie in der Akut- und Notfallmedizin [2]. Sie beinhalten hochmolekulare Substanzen wie Proteine oder Polysaccharide, die Wasser durch ihren

Die in dieser Publikation verwendeten Personen- und Berufsbezeichnungen treten der besseren Lesbarkeit halber nur in einer Form auf, sind aber natürlich gleichwertig auf beide Geschlechter bezogen. kolloidosmotischen Druck binden und das Gefäßsystem nicht verlassen können, sodass bis zu $100 \%$ der infundierten Menge intravasal zur Verfügung stehen.

Demgegenüber handelt es sich bei kristalloiden Infusionslösungen um Elektrolytlösungen, die keinen kolloidosmotischen Druck aufbauen können, da ihnen Makromoleküle fehlen und die Elektrolyte nur zu $25 \%$ im Intravasalraum verbleiben und $\mathrm{zu} 75 \%$ in den Extravasalraum umverteilt werden [3].

Um denselben Volumeneffekt zu erzielen, muss dementsprechend etwa die vierfache Menge an Kristalloiden infundiert werden $[4,5,6,7]$. Die damit einhergehende Gefahr der interstitiellen Überwässerung ist auch der entscheidende Nachteil einer Volumensubstitution mit Kristalloiden [8].

Mehrere Studien haben gezeigt, dass eine inadäquate Flüssigkeitsüberladung vermehrt Komplikationen induziert, u. a. durch eine generalisierte Ödembildung mit verzögerter Normalisierung der Darmfunktion, interstitielle Ödembildung mit Verlängerung der Sauerstoffdiffusionsstrecke und Sauerstoffmangelversorgung der Gewebe, Lungenödem und/oder abdominelles Kompartmentsyndrom $[9,6,10]$. Eine positive Flüssigkeitsbilanz ist darüber hinaus mit einer erhöhten Mortalität verbunden $[11,12]$. Andererseits ist auch ein nicht ausreichender Volumenersatz für den Patienten nachteilig [13, 14, 15].

Daher wurde in den letzten Jahren v. a. in der Chirurgie immer häufiger eine zielwertorientierte Volumentherapie angewandt, die sich nicht an absoluten Volumenmengen, sondern an der Optimierung von Kreislaufvariablen wie Schlagvolumen und Pulsdruck orientiert.

Unter dem Einfluss der jüngsten Entwicklungen in Hinblick auf Empfehlungen zum Einsatz von HES-Präparaten in der Intensivmedizin und bei elektiven Eingriffen sollen im vorliegenden Expertenstatement im ersten Teil die verschiedenen Aspekte einer rationalen patientenorientierten Volumenersatztherapie kurz dargestellt werden. Der zweite Teil des Statements ist die Zusammenfassung eines Expertenmeetings, bei dem österreichische Experten die präklinische und klinische Praxis der Volumentherapie anhand verschiedener Themenbereiche diskutierten.

\section{Volumenersatzmittel}

\section{Kolloide}

Künstliche Kolloidlösungen enthalten kolloidale Makromoleküle wie Kohlen- 
hydrate (Hydroxyethylstärke, Dextrane) oder Proteine (Gelatine oder humanes Albumin). Dextran spielt wegen der potenziellen Nebenwirkungen (renale Effekte, Beeinträchtigung der Hämostase, allergische Reaktionen, Notwendigkeit einer Haptenprophylaxe) in Mitteleuropa keine Rolle mehr.

Hydroxyethylstärke (HES) besteht aus Amylopektin (Mais, Kartoffeln), an dessen Glukosegruppen Hydroxyethylgruppen angefügt werden. HES wird enzymatisch gespalten und entweder metabolisiert oder durch das retikuloendotheliale System aus dem intravasalen Raum entfernt.

HES-Lösungen sind durch drei verschiedene Kriterien charakterisiert:

1. das Molekulargewicht, hier stehen

Lösungen zwischen 70 und $450 \mathrm{kDal}$ ton zur Verfügung,

2. den Substitutionsgrad, der die Anzahl der Hydroxyethylgruppen pro Glukosemolekül angibt (z. B. 0,4),

3. die Stelle im Glukosemolekül, an der eine Hydroxyethylgruppe angelagert wurde (C2 oder C6), woraus sich der Substitutionstyp ergibt, bezeichnet als das C2/C6-Hydroxyethylierungsverhältnis [16].

In Österreich stehen derzeit nur noch niedermolekulare HES-Präparationen der dritten Generation (130/0,4 bzw. 130/0,42) zur Verfügung.

$\mathrm{Zu}$ den wichtigsten Nebenwirkungen der HES-Lösungen gehören wie bei Dextran ein Coating-Effekt mit Abnahme der Thrombozytenadhäsivität sowie Veränderungen der plasmatischen und zellulären Blutgerinnung [17, 18]. Die Beeinträchtigung des Gerinnungssystems und der Thrombozytenfunktion ist umso geringer, je kleiner das Molekulargewicht, der Substitutionsgrad und das C2/C6Verhältnis sind. Am wenigsten ausgeprägt scheinen diese Nebenwirkungen bei niedermolekularem HES mit niedrigem Substitutionsgrad (z. B. HES 130/0,4 oder HES 70/0,5) zu sein [19, 20]. Niedermolekulare Präparate werden zum größten Teil renal eliminiert und nur zu einem sehr kleinen Teil im retikuloendothelialen System (RES) eingelagert [16]. Der Unterschied dieser Präparate im Vergleich zu mittel- oder hochmolekularen
Hydroxyethylstärken liegt in der kürzeren intravasalen Volumenwirkung in der Größenordnung von 2-3 Stunden.

Gelatine wird durch Hydrolyse aus Kollagen von Rinderhaut und Rinderknochen gewonnen. Als Volumenersatz bietet es gegenüber HES keine entscheidenden Vorteile: Der Volumeneffekt liegt nur bei rund $50 \%$ [21]. Das Risiko für anaphylaktische Reaktionen ist um eine Vielfaches höher als unter Humanalbumin [22, 23]. Gelatine beeinträchtigt die Hämostase bzw. Koagulation (erhöhte Blutviskosität, reduzierte Blutgerinnung) und verlängert so die Blutungszeit [24, 25, 26, 27]. Bei akuter Hämodilution im Rahmen einer kardiopulmonalen Bypassoperation ist die Gabe von HES 130/0,4 gegenüber Gelatine nicht nur in Hinblick auf eine verbesserte und raschere mikrovaskuläre Perfusion überlegen [28], sondern auch bzgl. Der Reduktion von Entzündungsmarkern (z. B. IL-8) und Erhöhung antiinflammatorischer Marker (z. B. Il-10) [29].

Die Gewinnung von Gelatine aus bovinem Rohmaterial ist zwar einem aggressiven Herstellungsverfahren unterworfen, dennoch kann die Übertragung des Erregers der bovinen spongiformen Enzephalopathie (BSE) grundsätzlich nicht vollständig ausgeschlossen werden [30, 31].

Obwohl Gelatine bereits seit rund 100 Jahren als Infusionslösung angewendet wird, ist die Datenlage mit der von HES kaum vergleichbar, da mit Gelatine keine großen kontrollierten Studien durchgeführt wurden, die ein Statement „Gelatine ist sicher" unzweifelhaft rechtfertigen könnten [32, 33]. Nicht zuletzt aufgrund von Sicherheitsbedenken ist Gelatine in den USA bereits seit 1978 nicht mehr zugelassen [34].

\section{Kristalloide}

Als Kristalloide werden Elektrolytlösungen oder niedermolekulare Kohlenhydratlösungen bezeichnet. Sie unterscheiden sich in Hinblick auf Osmolarität, kolloidosmotischen Druck, Elektrolytgehalt und $\mathrm{pH}$-Wert. Unbalancierte Kristalloide wie die fälschlicherweise als ,physiologisch" bezeichnete 0,9\%ige Natriumchlorid-(NaCl-)Lösung werden in Europa kaum mehr als Volumenersatzmittel verwendet, da der unphysiologisch hohe Chloridgehalt von $154 \mathrm{mmol} / \mathrm{l}$ mit Hyperchlorämie, Azidose oder Nierenfunktionsstörungen vergesellschaftet ist [35]. Speziell bei intensivmedizinischen Patienten wurden mehr hyperchlorämische Azidosen bzw. eine höhere Inzidenz an Nierenversagen mit Dialysepflichtigkeit berichtet $[36,37]$.

Demgegenüber wurden in der rezenten SPLIT-Studie bei kritisch Kranken keine nachteiligen Effekte von $\mathrm{NaCl}$ im Vergleich $\mathrm{zu}$ einem balancierten Kristalloid (Plasmalyte 148) auf die Nierenfunktion beobachtet, sofern nicht mehr als zwei Liter (Median) innerhalb von drei Tagen verabreicht werden [38]. Allerdings erhielten mehr als $60 \%$ der Patienten bereits vor Randomisierung ein balanciertes Kristalloid, das meiste Volumen wurde am Tag der Randomisierung verabreicht (NaCl: $1694 \pm 292$; Plasmalyte: $1711 \pm 1385)$. Die durchschnittlich verabreichte Kristalloidmenge betrug während des gesamten ICU-Aufenthalts allerdings nur zwei Liter, sodass sich aufgrund dieser Studie am Risiko-Nutzen-Profil von $\mathrm{NaCl}$ kaum etwas ändern dürfte [39]. Zudem wussten am Ende der Studie zwei Drittel der Ärzte trotz doppelter Verblindung, welches Kristalloid sie infundiert hatten, da es in der NaClGruppe vermehrt zu Hyperchlorämien und metabolischen Azidosen kam und aufgrund dieser Beobachtungen auf die Gabe von $\mathrm{NaCl}$ geschlossen wurde, auch wenn die Chloridwerte nicht dokumentiert wurden [38].

Seit vielen Jahren werden in der Chirurgie bevorzugt Vollelektrolytlösungen wie Ringer-Laktat eingesetzt. Da die $\mathrm{Zu}-$ fuhr von Laktat bei Patienten mit bereits erhöhten Laktatwerten nicht sinnvoll ist, enthalten einige kristalloide Lösungen stattdessen Acetat oder Malat, die wie Laktat zu Bikarbonat metabolisiert werden, wodurch eine Dilutionsazidose vermieden werden kann [40]. Während Acetat unabhängig von der Leber verstoffwechselt wird, ist der vorwiegende metabolische Weg für infundiertes Laktat mit bis $\mathrm{zu} 70 \%$ die intrahepatische Glukoneogenese [41]. Dies ist insbesondere bei Sepsispatienten von prognostischer Bedeutung, bei denen die hepatische Laktat-Clearance aufgrund des 
Schockzustandes beeinträchtigt ist [42, $43,44]$.

Die hepatische Metabolisierung von Laktat benötigt außerdem überproportional viel Sauerstoff, sodass pro Liter Ringer-Laktat der Sauerstoffbedarf des Patienten für 7 min verdoppelt wird [45].

Im Gegensatz zu Kolloiden verteilen sich Kristalloide nicht nur intravasal, sondern im gesamten Extrazellularraum, demnach muss für einen vergleichbaren unmittelbaren Volumeneffekt deutlich mehr Kristalloid als isoonkotisches Kolloid verabreicht werden.

Jacob et al. [6] untersuchten mit Hilfe der direkten Bestimmung des menschlichen Blutvolumens durch DoubleTracer-Techniken vor und nach Infusion die intravasalen Volumeneffekte von Ringer-Laktat, $5 \%$ Humanalbumin und $6 \%$ HES 130/0,4. Dabei war der mittlere Volumeneffekt von Ringer-Laktat kleiner als $20 \%$. Über $80 \%$ belasteten als Ödem das Interstitium. In dieser Studie war es faktisch nicht möglich, bei relevanter Blutung ( $>11$ in $30 \mathrm{~min}$ ) mit Ringer-Laktat die Normovolämie zu erhalten. Dies war bei der indikationsgerechten Verwendung isoonkotischer Kolloide anders: 5\% Humanalbumin, $6 \%$ HES $130 / 0,4$ und $6 \%$ HES $200 / 0,5$ zeigten hier mittlere Volumeneffekte von 82-98\% [46, 47].

Unter Berücksichtigung ihrer unterschiedlichen pharmakologischen Eigenschaften sind kristalloide Lösungen idealerweise in balancierter Form - für den Ausgleich des präoperativen Defizits und des intraoperativen Erhaltungsbedarfs indiziert, während kolloidale Lösungen bei Zeichen einer Hypovolämie gegeben werden sollten $[48,49,50]$.

Dies wird durch die Ergebnisse von experimentellen und klinischen Untersuchungen gestützt, in denen unterschiedliche Kolloide im Vergleich zu Kristalloiden nicht nur Verbesserungen im Hinblick auf Volumenwirksamkeit gezeigt haben, sondern auch auf eine Normalisierung des kolloidosmotischen Drucks (KOD), der Inzidenz von interstitiellen Ödemen, der Leukozyten-EndothelInteraktionen und der Gewebeoxygenierung hinweisen $[51,49,52,53,54,55,56]$.

In einer rezenten Studie erhöhte HES 130/0,4 den KOD in gesunden Freiwil-

Wien klin Mag 2016 · 19:154-169 DOI 10.1007/s00740-016-0139-9

๑ Der/die Autor(en) 2016. Dieser Artikel ist eine Open-Access-Publikation.

W. Fartek · G. Gemes · M. Hiesmayr · B. Kabon · S. Kozek-Langenecker · R. Likar · G. Luckner · W. Mitterndorfer $\cdot$ C. Torgersen

\section{Stellenwert von Hydroxyethylstärkelösung in der Volumenersatztherapie. Ergebnisse eines österreichischen Expertentreffens}

\section{Zusammenfassung}

Die intravasale Volumentherapie ist eine der häufigsten therapeutischen Maßnahmen im periinterventionellen Setting. Sie wird insbesondere bei Patienten mit akuter Hypovolämie, bei hämodynamischer Instabilität oder Schock durchgeführt, um ein ausreichendes intravasales Volumen und Sauerstoffangebot sicherzustellen und konsekutiv eine adäquate Pumpleistung des Herzens, Gewebeperfusion, Gewebeoxygenierung und Organfunktion zu erreichen. Kolloide haben gegenüber Kristalloiden den Vorteil der höheren intravasalen Volumenwirksamkeit; zur Indikationsstellung und Dosierungssteuerung empfehlen Fachgesellschaften ein vorlastbasiertes hämodynamisches Monitoring. Internationale Studien mit relevanten Limitationen, z.B. durch insensitives Hypovolämiemonitoring mit ungezielter Dosierung der Infusionslösungen, haben weltweit eine kontroversielle Diskussion zum Stellenwert von HES ausgelöst und eine Zulassungseinengung bewirkt. In diesem Artikel wird die Meinung österreichischer Kliniker zum Volumenmonitoring im Allgemeinen und zu HES im Speziellen dargestellt. Insgesamt wird derzeit HES reflektiert, restriktiv und gezielt zur Korrektur der Hypovolämie bei sämtlichen Patientenkollektiven bundesweit eingesetzt. Rekapillarisierungszeit, Pulskonturanalyse und Echokardiographie sind die am meisten benutzten Methoden zum Volumenmonitoring bei großen Operation, bei Patienten mit Komorbiditäten bzw. hamodynamischer Instabilität.

\section{Schlüsselwörter}

Volumentherapie · Hypovolämie · Kolloid . Hydroxyethylstärkelösung

\section{Importance of Hydroxyethyl Starch in Volume Replacement Therapy. Results of an Austrian Expert Meeting}

\section{Abstract}

Volume substitution is one of the most frequent therapeutical intervention in the perioperative setting. In patients with acute hypovolaemia, haemodynamic instability, or shock, volume therapy is required for restoring intravasale volume and oxygen delivery, tissue perfusion and oxygenation, and for protecting organ function. Compared with crystalloids colloidal solutions offer the advantage of a higher volume efficacy; for inidcating and dosing colloids scientific societies recommend preload-based haemodynamic monitoring. International studies with relevant limitations, e. g. because of inappropriate monitoring for hypovolaemia with resulting non-individualized dosing of the infusion solution promoted globally controversial discussion on the role of HES. The present article summarizes the opinion of Austrian clinicians on volume monitoring in general as well as on the importance of HES. Under the bottom line, there is currently nationwide a restricitve and targeted use of HES for correcting hypovolaemia in a broad spectrum of perioperative patient populations. Capillary refill, pulse curve analyses, and echocardiography are the most frequently used methods for volume monitoring in major surgery, in patients with co-morbidities or haemodynamic instability.

\section{Keywords}

Volume therapy · Hypovolaemia · Colloid Hydroxyethylstarch ligen, während er unter Ringer-Acetat abnahm [57]. In einem Tiermodell zu kontrolliertem hämorrhagischen Schock konnte mit HES 130/0,4 die Hämodynamik nach einer Blutung viermal rascher als mit Ringer-Laktat und mit signifikant weniger Volumen stabilisiert werden [58]. Zudem waren in einer aktuellen Analyse der 6S-Studie unter HES 130/0,42-Gabe Biomarker eines Endothelschadens und einer Gerinnungsstörung (i.e. lösliches Thrombomodulin, Syndecan 1, Plasminogen-AktivatorInhibitor, löslicher CD40-Ligand, Gewe- 
beplasminogenaktivator) versus RingerAcetat deutlich reduziert [59].

Eine adäquate Infusionstherapie sollte bedarfsorientiert und unter Berücksichtigung physiologischer Grundprinzipien durchgeführt werden. Um eine Hypo- und Hypervolämie zu vermeiden, sollte der tatsächliche intravasale Volumenmangel durch ein geeignetes Kolloid und der basale Flüssigkeitsbedarf durch eine kontinuierliche Infusion balancierter Kristalloide ausgeglichen werden.

\section{Physiologischer Exkurs: die Funktion der endothelialen Glykokalyx}

Das Konzept einer kolloidbasierten Volumenersatztherapie wird auch durch experimentelle Studienergebnisse zur Funktion des sogenannten „endothelial surface layer" (ESL) gestützt [60]. Er besteht aus der Glykokalyx, einer bis $\mathrm{zu} 1000 \mathrm{~nm}$ großen Struktur auf der endothelialen Oberfläche mit gebundenen Plasmaproteinen, die eine wichtige Barrierefunktion zwischen intravasalem und interstitiellem Kompartiment des Extrazellularraums einnimmt [61, 62, 63]. Als vaskulär-endotheliale Barriere verhindert die Glykokalyx trotz permanentem (Blut-)Druck einen quantitativ relevanten druckpassiven Abstrom von Proteinen in das Interstitium während kleine gelöste Substanzen, wie z. B. Elektrolyte nicht zurückgehalten werden und sich frei im gesamten Extrazellularraum verteilen können.

Wird die Glykokalyx zerstört, treten ungehindert hochmolekulare Plasmabestandteile und in der Folge auch Wasser in das Interstitium aus. Weitere potenzielle deletäre Folgen einer zerstörten endothelialen Oberfläche sind eine lokale und generalisierte Inflammation sowie eine kompromittierte Mikroperfusion.

Sowohl im perioperativen als auch im intensivmedizinischen Kontext korreliert die Zerstörung der Glykokalyx bei Patienten im septischen Schock mit dem Mortalitätsrisiko, nach Ischämie und Reperfusion mit der Dauer und dem Ausmaß der Ischämie, und ist darüber hinaus bei Patienten nach Trauma ein unabhängiger Prädiktor für die Mortalität. Klinische Studien haben gezeigt, dass auch

Hier steht eine Anzeige. Springer 
Tab. 1 Empfehlungen der EMA in Hinblick auf den Einsatz von HES-Lösungen (Auszug) [78]

Aufgrund des Risikos der Nierenschädigung und der Mortalität dürfen HES-Lösungen bei Patienten mit Sepsis, mit Verbrennungen oder bei intensivpflichtigen Patienten nicht mehr verwendet werden

HES-Lösungen sollten nur zur Behandlung von Hypovolämie aufgrund akuten Blutverlusts eingesetzt werden, wenn Kristalloide allein nicht als ausreichend angesehen werden

Für Patienten, die sich chirurgischen Eingriffen unterziehen, und für Traumapatienten liegen keine zuverlässigen Daten zur langfristigen Sicherheit vor. Der erwartete Nutzen der Behandlung sollte sorgfältig gegen die Unsicherheiten im Hinblick auf die langfristige Sicherheit abgewogen werden, und es sollten andere Behandlungsmöglichkeiten in Betracht gezogen werden. Weitere Studien mit HES-Lösungen bei Patienten mit Trauma sowie Patienten, die sich einer elektiven Operation unterziehen, werden durchgeführt

HES-Lösungen sollten über den kürzestmöglichen Zeitraum und in der niedrigsten wirksamen Dosierung angewendet werden. Die Behandlung sollte sich an einer kontinuierlichen hämodynamischen Überwachung orientieren, wobei die Infusion beendet werden sollte, sobald die angestrebten hämodynamischen Zielwerte erreicht sind

Bei Patienten mit Niereninsuffizienz oder unter Nierenersatztherapie sind HES-Lösungen nun kontraindiziert

eine Hypervolämie zur Abnahme des Volumens des „endothelial surface layers“ führt [64].

Die Intaktheit der Glykokalyx ist durch verschiedene Faktoren gefährdet, die häufig bei kritisch kranken Patienten oder infolge eines Traumas anzutreffen sind. So kommt es beispielsweise durch hohe Konzentrationen proinflammatorischer Zytokine und Ischämie/ Reperfusion zu einer Degradation der Glykokalyx [61, 65, 66]. Darüber hinaus führt eine intravasale Hypervolämie durch Dehnung der Herzvorhöfe zur Freisetzung natriuretischer Pepetide. Diese und vor allem ANP (atriales natriuretisches Peptid) aktivieren Metalloproteasen, die die endotheliale Glykokalyx degradieren. Es kommt zu einem vermehrten Flüssigkeitsabstrom ins Interstitium [67]. Zudem gefährdet eine akute präoperative Hypervolämie durch Gabe von Flüssigkeitsboli die Integrität des ESL [21].

Eine intakte endotheliale Glykokalyx ist zentraler Bestandteil einer physiologisch wirksamen vaskulären Barriere. Wird sie durch Trauma, Chirurgie oder Hypervolämie degradiert, kommt es zu einem proteinreichen Flüssigkeitsshift in den interstitiellen Raum.

\section{Rekapitulieren der großen klinischen Studien: Kolloide zur initialen Stabilisierung}

Während Studien und entsprechende Metaanalysen im perioperativen Set- ting die erfolgreiche Behandlung einer Hypovolämie mit modernen HES-Präparaten belegen und deren Stellenwert und Sicherheit bei chirurgischen $\mathrm{Pa}$ tienten außer Frage steht $[68,69,70$, $71,72,73]$, führten Untersuchungen an kritisch kranken, v. a. septischen Patienten aufgrund ihrer Studienprotokolle und Ergebnisse in den letzten Jahren zu heftigen Diskussionen und trugen eher zur Verunsicherung als zur Klärung der Situation bei. Kaum ein anderes Thema der Intensivmedizin wurde so emotional diskutiert wie das Volumen- und Flüssigkeitsmanagement [68].

Ausgangspunkt der heftigen Diskussionen war die 2008 publizierte VISEPStudie [74], gefolgt von der 6S-Studie [75] und CHEST [76], in denen unter HESPräparaten versus Ringer-Laktat/Acetat bei septischen bzw. intensivpflichtigen Patienten eine erhöhte Rate an Nierenersatzverfahren beobachtet wurde. Die Ergebnisse dieser Studien führten $\mathrm{zu}$ einem Pharmakovigilanzverfahren der europäischen Zulassungsbehörde EMA, die in der Folge den Einsatz von HES bei kritisch kranken Patienten deutlich einschränkte. Im Oktober 2013 gab der Ausschuss für Risikobewertung im Bereich der Pharmakovigilanz (PRAC) die Empfehlung ab, HES bei Sepsis, Brandverletzungen und intensivpflichtigen $\mathrm{Pa}$ tienten aufgrund eines erhöhten Risikos für Nierenversagen und Mortalität nicht mehr anzuwenden [77]. Die Empfehlung mündete am 19. Dezember 2013 in einem endgültigen rechtsverbindlichen
Beschluss der Europäischen Kommission mit Gültigkeit in der gesamten Europäischen Union (• Tab. 1; [78]).

Inwieweit diese Maßnahmen wissenschaftlich gerechtfertigt sind, bleibt dahingestellt, Tatsache ist jedoch, dass eine genauere Analyse der Studien zahlreiche Mängel und Protokollverletzungen zutage bringt.

In der VISEP-Studie $(n=537$, kochsalzbasiertes $10 \%$ HES 200/0,5 versus Ringer-Laktat) wurde bei Patienten mit septischem Schock eine „alte“ hyperonkotische HES-Lösung verwendet, die zu Studienbeginn bereits von isoonkotischen Lösungen mit einem niedrigeren Molekulargewicht von $130 \mathrm{kDa}$ als Standard abgelöst waren, da mittlerweile bekannt war, dass hyperonkotische größermolekulare HES-Präparate Nierenfunktionsstörungen begünstigen können [79].

Das hyperonkotische HES-Präparat wurde innerhalb der ersten $24 \mathrm{~h}$ im Median um $60 \%$, maximal sogar um $700 \%$ gegenüber der in der Fachinformation als Tagesmaximaldosis festgehaltenen Menge überdosiert. Die initiale hämodynamische Stabilisierung wurde in beiden Behandlungsgruppen meist durch ein Kolloid durchgeführt (58\% der Patienten in der Kristalloid-Gruppe), und zwar noch bevor die Patienten in die Studie eingeschlossen wurden [74, Appendix]. Die Randomisierung der Patienten in die Kolloid- und Kristalloid-Gruppe erfolgte erst bis zu $24 \mathrm{~h}$ nach Diagnosestellung des septischen Schocks, sodass Patienten, die bereits stabilisiert waren, ein nicht mehr zeitgemäßes Kolloid über einen längeren Zeitraum und außerhalb der Indikation erhielten.

Bei Randomisierung lagen daher die medianen Werte für den zentralvenösen Druck (ZVD) bei $12 \mathrm{~mm} \mathrm{Hg}$, die zentralvenöse Sauerstoffsättigung $\left(\mathrm{SzvO}_{2}\right)$ bei $74 \%$ und Serumlaktat bei $2,2 \mathrm{mmol} / \mathrm{l}$ und befanden sich somit bereits vor Studienbeginn im Bereich der von der Surviving Sepsis Campaign empfohlenen Zielwerte einer angestrebten Volumentherapie [80].

In der $6 S$-Studie ( $n=798$, balanciertes $6 \%$ HES 130/0,42 versus Ringer-Acetat) wurden $42 \%$ der Patienten aufgrund ihres septischen Schockzustandes bereits vor Studieneinschluss mit bis zu 1 Liter 
HES behandelt [75, Appendix]. Dementsprechend lagen - ähnlich wie in VISEP - die medianen Werte zu Studienbeginn für den ZVD bei $10 \mathrm{~mm} \mathrm{Hg}$, die $\mathrm{SzvO}_{2}$ bei $75 \%$ und Serumlaktat bei $2,0 \mathrm{mmol} / \mathrm{l}$, sodass die Indikation für eine (weitere) Volumentherapie äußerst fraglich erscheint.

Darüber hinaus wurde bei $36 \%$ der Patienten in der HES-Gruppe (35\% in der Ringer-Acetat-Gruppe) ein Nierenversagen diagnostiziert. Diese Patienten hätten gemäß Fachinformation aufgrund der Kontraindikation kein HES-Präparat erhalten dürfen. Zudem brachen $216 \mathrm{~Pa}$ tienten in beiden Gruppen (26\%) die Flüssigkeitstherapie ab und $32 \%$ der Patienten in der Kristalloid-Gruppe erhielten zusätzlich Kolloide während des Studienverlaufs [68].

Demgegenüber zeigten die CHESTStudie [76] mit einem heterogenen intensivmedizinischen Patientenkollektiv ( $n=7000)$ und die CRYSTMAS-Studie (196 Patienten mit septischem Schock) [81], in denen $6 \%$ HES 130 mit isotoner Kochsalzlösung verglichen wurde, bei septischen Patienten keine klinisch relevanten negativen Effekte unter HES [68].

Zusammenfassend konnte sowohl in der VISEP-Studie als auch in der 6SStudie die initiale hämodynamische Stabilisierung des septischen Schocks - gemessen an der Zielerreichung der Kriterien der Surviving Sepsis Campaign erfolgreich auch mit HES behandelt werden. Allerdings fehlte in beiden Studien der Nachweis der Hypovolämie durch ein geeignetes Volumenmonitoring, da nur druckbasierte hämodynamische Parameter gemessen wurden.
Dieses Vorgehen steht im Einklang mit der viel zitierten Studie von Rivers und Mitarbeitern [82], in der eine frühzeitige aggressive Optimierung der kardialen Vorlast und der zentralvenösen Sauerstoffsättigung innerhalb von sechs Stunden nach Diagnose mit einer deutlichen Verbesserung des klinischen Ergebnisses und des Überlebens bei Patienten mit schwerer Sepsis und septischem Schock verbunden war. Die frühe hämodynamische Stabilisierung gilt seither als State of the Art [80]. Diese entscheidende Zeitspanne wurde jedoch in beiden Studien nicht berücksichtigt, sondern lediglich der weitere Verlauf nach erfolgter Stabilisierung auf der Intensivstation.

Die beiden Studien VISEP und 6 S belegen, dass die Weiterverwendung von HES als Erhaltungstherapie anstelle eines Kristalloids nach initialer Stabilisierung kontraindiziert ist.

Andererseits konnten Annane et al. [83] in der großen randomisierten CRISTAL-Studie mit über 2800 Patienten mit hypovolämischem Schock zeigen, dass die frühzeitige Gabe von Kolloiden (meist $6 \%$ HES 130/0,4) gegenüber Kristalloiden mit einer niedrigeren 90-Tage-Mortalität assoziiert ist. Dies galt auch für die Patienten mit Sepsis (54\%). Darüber hinaus zeigte sich ein signifikanter Unterschied in den Überlebenstagen ohne Beatmung und ohne Katecholamine am 7. Tag und am 28. Tag, ein signifikant niedriger Volumenbedarf am Tag 7 und ein niedrigeres Risiko für eine Nierenersatztherapie zugunsten der Kolloidgabe.

In der CRISTAL-Studie profitierten Patienten im hypovolämen, zum Teil septischen Schock von einer frühzeitigen hä- modynamischen Stabilisierung durch die Kolloidgabe.

\section{Geeignete Parameter zum Volumenmonitoring}

Vor der Substitution einer Flüssigkeit sollte gemäß rezenter S3-Leitlinie die Diagnose des Volumenmangels anhand der Anamnese, ergänzt durch Laborparameter wie Laktatkonzentration, $\mathrm{SzvO}_{2}$, Hämatokrit (HKT) und Basenüberschuss gestellt werden [84].

Die Steuerung einer Volumenersatztherapie sollte immer zielorientiert sein. Nur mit dem geeigneten Monitoring kann die Indikation und die Dosis eruiert werden. Im Vordergrund steht dabei die Sicherstellung des Sauerstoffangebots durch Optimierung der kardialen Funktion - Vorlast, Kontraktilität und Nachlast. Durch das Monitoring der kardialen Vorlast sollen eine perioperative Hypovolämie und ihre negativen Auswirkungen auf das Herzzeitvolumen (HZV) verhindert werden und gleichzeitig einer unangepasst hohen Flüssigkeitszufuhr und den daraus resultierenden Risiken vorgebeugt werden [85].

Dabei sind flussbasierte, dynamische Parameter wie Schlagvolumenvarianz (SVV) und Pulsdruckvarianz (PPV) besser geeignet als statische druckbasierte, da mehrfach gezeigt werden konnte, dass sich die aktuelle kardiale Vorlast eines Patienten anhand der traditionellen statischen Zielgrößen (ZVD, pulmonalarterieller Okklusionsdruck, PAOD) nicht adäquat abbilden lässt [86, 87, 88]. Folglich sprechen sich die aktuellen Leitlinien der European Society

\section{Fachkurzinformationen}

Voluven (HES 130/0,4) 6 \% - Infusionslösung. Qualitative und quantitative Zusammensetzung: $1000 \mathrm{ml}$ Infusionslösung enthalten: Poly(O-2-hydroxyethyl)stärke (Ph.Eur.) 60,00 g (molare Substitution: 0,38-0,45, mittleres Molekulargewicht: $130.000 \mathrm{Da}$ ) (hergestellt aus Wachsmaisstärke), Natriumchlorid 9,00 g. Elektrolyte: $\mathrm{Na}^{+} 154 \mathrm{mmol}$, Cl-154 mmol. Theoretische Osmolarität: $308 \mathrm{mosmol} / \mathrm{l}$. pH-Wert: 4,0-5,5. Titrationsazidität: $<1,0 \mathrm{mmol} \mathrm{NaOH/l.Vollständige} \mathrm{Auflistung} \mathrm{der} \mathrm{sonstigen} \mathrm{Bestandteile} \mathrm{siehe} \mathrm{Abschnitt} \mathrm{6.1.} \mathrm{Anwendungsgebiete:} \mathrm{Behandlung} \mathrm{einer} \mathrm{Hypovolämie} \mathrm{bei} \mathrm{akutem} \mathrm{Blut-}$ verlust, wenn Kristalloide alleine als nicht ausreichend erachtet werden (siehe Abschnitte 4.2, 4.3 und 4.4). Gegenanzeigen: Überempfindlichkeit gegen die Wirkstoffe oder einen der in Abschnitt 6.1 genannten sonstigen Bestandteile; Sepsis; Verbrennungen; Nierenfunktionsstörung oder Nierenersatztherapie; intrakranielle oder zerebrale Blutung; kritisch kranke Patienten (in der Regel Patienten, die auf die Intensivstation aufgenommen werden müssen); Hyperhydratation; Lungenödem; Dehydratation; schwere Hypernatriämie oder schwere Hyperchlorämie, schwere Leberfunktionsstörundie auf die Intensivstation aufgenommen werden müssen); Hyperhydratation; Lungenodem; Dehydratation; schwere Hypernatriamie oder schwere Hyperchloramie, schwere Leberfunktionsstörun-
gen; dekompensierte Herzinsuffizienz; schwere Gerinnungsstörung; organtransplantierte Patienten. Pharmakotherapeutische Gruppe: Plasmaersatzmittel und Plasmaproteinfraktionen. ATC-Code: B05AA07. Liste der sonstigen Bestandteile: Natriumhydroxid, Salzsäure, Wasser für Injektionszwecke. Rezeptpflicht/Apothekenpflicht: Rezept- und apothekenpflichtig. Inhaber der Zulassung: Fresenius Kabi Austria GmbH, A-8055 Graz. Stand der Information: April 2016.

Volulyte 6 \% Infusionslösung. Qualitative und quantitative Zusammensetzung: $1000 \mathrm{ml}$ Infusionslösung enthalten: Poly(0-2-hydroxyethyl)stärke (Ph.Eur.) 60,00 g (molare Substitution: 0,38-0,45, mittleres Molekulargewicht: $130.000 \mathrm{Da}$ ) (hergestellt aus Wachsmaisstärke), Natriumacetat-Trihydrat 4,63 g, Natriumchlorid 6,02 g, Kaliumchlorid 0,30 g, Magnesiumchlorid-Hexahydrat 0,30 g. Elektrolyte:

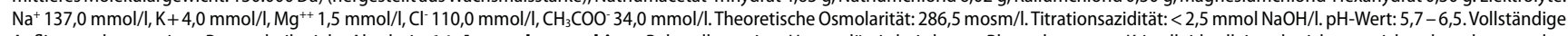
Auflistung der sonstigen Bestandteile siehe Abschnitt 6.1. Anwendungsgebiete: Behandlung einer Hypovolämie bei akutem Blutverlust, wenn Kristalloide alleine als nicht ausreichend erachtet werden (siehe Abschnitte 4.2, 4.3 und 4.4). Gegenanzeigen: Überempfindlichkeit gegen die Wirkstoffe oder einen der in Abschnitt 6.1 genannten sonstigen Bestandteile; Sepsis; Verbrennungen; Nierenfunktionsstörung oder Nierenersatztherapie; intrakranielle oder zerebrale Blutung; kritisch kranke Patienten (in der Regel Patienten, die auf die Intensivstation aufgenommen werden müssen); Hyperhydratation; Lungenödem; Dehydratation; schwere Hyperkaliämie; schwere Hypernatriämie oder schwere Hyperchlorämie; schwere Leberfunktionsstörungen; dekompensierte Herzinsuffizienz; schwere Gerinnungsstörung; organtransplantierte Patienten. Pharmakotherapeutische Gruppe: Plasmaersatzmittel und Plasmaproteinfraktionen. ATC-Code: B05AA07. Liste der sonstigen Bestandteile: Natriumhydroxid (zur pH-Wert Einstellung), Salzsäure (zur pH-Wert Einstellung), Wasser für Injektionszwecke. Rezeptpflicht/Apothekenpflicht: Rezept- und apothekenpflichtig. Inhaber der Zulassung: Fresenius Kabi Austria GmbH, A-8055 Graz. Stand der Information: Februar 2016. 
of Anesthesiology (ESA) zum Management perioperativer schwerer Blutungen gegen die alleinige Nutzung von ZVD und PAOD zur Steuerung der Volumentherapie und Optimierung der kardialen Vorlast und für dynamische Parameter sowie nichtinvasive Methoden zur Messung des HZV aus [89].

Die Volumenreagibilität sollte entweder durch Lagerungsmanöver (PLR, „passive leg raising“) oder mithilfe des SVV oder PPV gemessen werden, da weder ZVD noch PAOD in der Lage sind, mit ausreichender Sensitivität und Spezifität eine Volumenreagibilität vorherzusagen [90, 84, 87, 91, 92, 93].

Allerdings bedeutet Volumenreagibilität nicht, dass immer Volumen verabreicht werden muss bzw. werden darf. Voraussetzung dafür ist der klinische Nachweis einer Minderperfusion, d.h. wenn zwei oder mehr der folgenden Zeichen vorhanden sind:

- Hautmarmorierung oder schlechte periphere Perfusion (z. B. kalte Hände/Füße),

- Kaltschweißigkeit,

- Oligurie,

- verzögerte kapilläre Füllungszeit,

- erhöhtes Laktat (>2 mmol/L oder $>18 \mathrm{mg} / \mathrm{dL}$ ),

- erniedrigte zentral-/gemischtvenöse $\mathrm{SO}_{2}(<60 \%)$,

- Verwirrtheit/Unruhe.

Die Füllungsdrücke ZVD und PAOD sind zur Abschätzung der kardialen Vorlast nicht geeignet.

Druck ist kein Volumen.

Darüber hinaus sind ZVD und PAOD aufgrund der Invasivität des Messverfahrens auf Hochrisikoeingriffe beschränkt. Sie können aber als Stopp-Signal für eine weitere Volumenzufuhr genutzt werden $[94,84]$.

Im Gegenzug dazu bieten bereits nichtinvasive Technologien eine Vielfalt an kontinuierlichen dynamischen Parametern, die es ermöglichen, eine zielgerichtete Volumentherapie in der täglichen Routine durchzuführen - auch beim Patienten mit mittlerem Risiko [95]. Die perioperative Outcome-Verbesserung der zielgerichteten Volumentherapie mit nichtinvasiven Messmethoden ist klinisch bestätigt [96, 97].
Bei Intensivpatienten kann zur Untersuchung des Volumenstatus eine transthorakale Echokardiographie (TTE) erfolgen. Bei Patienten mit unklarer hämodynamischer Instabilität empfehlen die Leitlinien ebenfalls, eine Echokardiographie zur Diagnose durchzuführen. Dadurch kann bei diesen Patienten eine Vielzahl von relevanten Differenzialdiagnosen (Perikarderguss und -tamponade, akute Rechtsherzbelastung als Hinweis für eine Lungenarterienembolie, eingeschränkte Pumpfunktion, Klappenvitium etc.) eingegrenzt werden [84]. Insbesondere beim kardiogenen Schock, aber auch bei allen anderen Schockformen, sind TTE und transösophageale Echokardiographie (TEE) zentraler Bestandteil in der Diagnostik und Differenzialdiagnostik [98].

Zur Abschätzung des Volumenbedarfs bzw. der Volumenreagibilität sollen funktionelle hämodynamische Parameter genutzt werden; diese sollen nicht isoliert betrachtet werden, sondern stets unter Einbeziehung von Anamnese und klinischem Untersuchungsbefund [84].

\section{Steigerung der Anämietoleranz durch Volumentherapie}

Folgesymptome einer relevanten Anämie entstehen durch globale oder regionale Sauerstoffdefizite. Solche anämiebedingten Symptome, wie z. B. STStreckenveränderungen, Delir und Niereninsuffizienz, treten eher bei Hypoals bei Normovolämie auf, eher bei hohem als bei niedrigem Sauerstoffbedarf. Entsprechend können (oberhalb des individuellen, kritisch niedrigen Hämoglobinwerts) therapeutische Interventionen die Empfindlichkeit gegenüber einer bestehenden Anämie herabsetzen, d. h. eine Anämietoleranz herbeiführen. Der österreichische interdisziplinäre Konsensus zum patientenorientierten Blutmanagement (POBM) empfiehlt zur Erhöhung der Anämietoleranz situationsangepasst und individualisiert anästhesiologisch-intensivtherapeutische Maßnahmen [99] wie die Erhöhung des Sauerstoffangebots über Optimierung des Herzzeitvolumens (inklusive Volumentherapie, Vasopressoren, Inotropika, Vasodilatatoren). Kolloide können dazu genutzt werden, bei bestehender Anämie eine Hypovolämie zu korrigieren und damit Fremdbluttranfusionen oberhalb eines kritischen physiologischen Hämoglobintriggers [100] zu vermeiden. Die Kompensationsmechanismen einer normovolämischen Anämie können auch bei Säuglingen, Kindern, alten Patienten, kardial vorerkrankten Patienten und $\mathrm{Pa}$ tienten unter chronischer $\beta$-RezeptorBlockade nachgewiesen werden [101].

Weitere Maßnahmen zur Anämietoleranzsteigerung sind die Optimierung der Beatmung (hyperoxisch, normokapnisch), Reduktion des Sauerstoffverbrauchs über therapeutische (milde) Hypothermie, adäquate Muskelrelaxation, Ausschalten von Stressreizen (mittels adäquater Sedierungs- bzw. Anästhesietiefe und Analgesie), Vermeidung bzw. Behandlung von schweren Erkrankungen wie z. B. Sepsis, Trauma und Herzerkrankung.

Postoperativ können auf der Bettenstation folgende allgemeinen Maßnahmen erwogen werden: Aufrechterhaltung der Normovolämie, Schmerztherapie, Weiterbehandlung von vorbestehenden und Vermeidung von sekundären Erkrankungen, Vermeidung von Medikamenteninteraktionen, die eine Anämie oder Blutung begünstigen, gegebenenfalls passive (anstatt aktive) Mobilisierung, Sauerstoffinsufflation [99]. Die postoperative Korrektur eines Eisenmangels kann in Erwägung gezogen werden, aber der Therapieeffekt ist verzögert zu erwarten.

Während einer Hypovolämie ist die Anämietoleranz des Körpers reduziert. Grundvoraussetzung für die effektive Kompensation der Verdünnungsanämie ist die Normovolämie.

\section{Geeignete Algorithmen zur Volumentherapie: „goal-directed therapy" (GDT)}

Im Kontext einer Volumentherapie erfolgt die zielgerichtete hämodynamische Therapie (GDT) gemäß einem vordefinierten Therapiealgorithmus mit Parametern, die direkte oder indirekte Informationen über fluss-, volumenoder stoffwechselbezogene Determinanten der Hämodynamik liefern, um durch 
Hier steht eine Anzeige.

\section{曾 Springer}




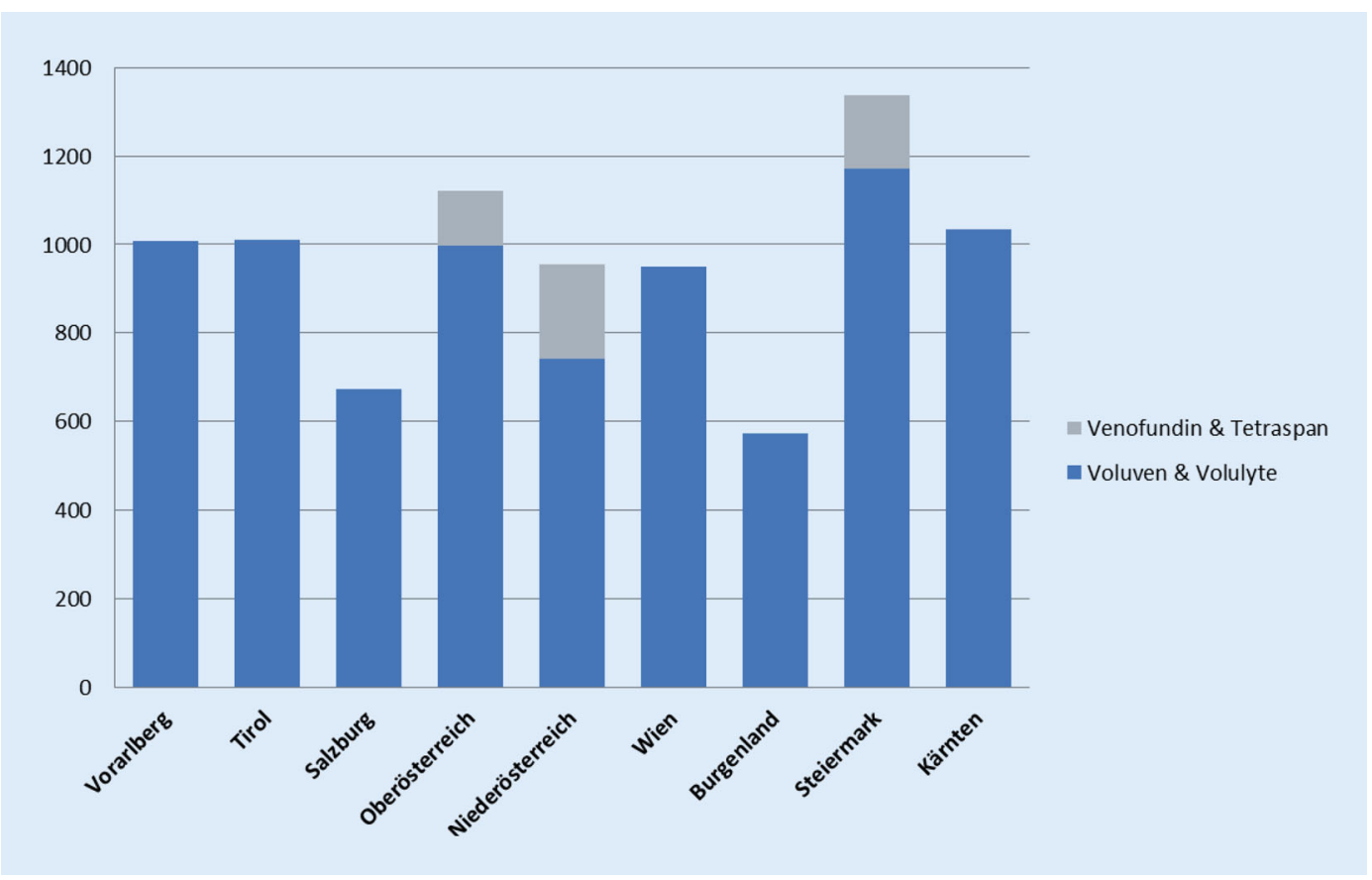

Abb. $1 \triangleleft$ Verwendete Einheiten HES im Jahr 2015 pro 100.000 Einwohner: Eine Einheit entspricht einerverkauften Dosis Hydroxyethylstärke, unabhängig von Behältnis und Volumen. Geschätzte Verteilung der IMS Health Marktforschung GmbH für 2015 von Tetraspan und Venofundin: Einwohnerzahlen 2015 laut bevölkerungsstatistischem Datenbanksystem der Statistik Austria (POPREG)

eine individuell angepasste Optimierung des Sauerstoffangebots die perioperative Morbidität und Mortalität zu senken.

Studien und Metaanalysen zeigen, dass eine GDT in Verbindung mit flussbasierten Parametern perioperativ die Komplikationsrate und auch die Mortalität senken kann [94, 102, 103, 104, 105, 106, 70].

Allerdings bestätigen nicht alle Studien einen signifikanten Benefit für chirurgische Patienten durch eine GDT im Vergleich zu den Kontrollgruppen mit konventioneller Versorgung [107, 108, 109]. Ein Cochrane-Review, der 38 Studien inkludierte, legt nahe, dass durch eine GDT die perioperative Mortalität nicht reduziert werden kann, wohl aber die Komplikationsrate und Länge des Spitalaufenthalts [110].

Eine der größten Studien, OPTIMISE, die 734 Hochrisikopatienten einschloss, die sich einem abdominalen Eingriff unterziehen mussten, erbrachte für die GDT durch Monitoring des HZV keinen signifikanten Vorteil im Hinblick auf die 30Tage-Mortalität $(p=0,07)$ oder schwere Komplikationen [111].

Welcher Algorithmus den höchsten Therapieeffekt erzielt, ist derzeit nicht geklärt. Ein systematischer Review identifizierte 81 Studien mit insgesamt 13.052 Patienten zur GDT, in denen 31 unter- schiedliche Zielparameter (Z) mit 22 verschiedenen Monitoring-Methoden (M) und letztlich 118 Z/M-Kombinationen zur Anwendung kamen [112]. Diese Untersuchung spiegelt die Vielzahl an Möglichkeiten wider, eine an unterschiedliche Patientenkollektive einerseits und den individuellen Patienten andererseits angepasste GDT anzuwenden.

Die GDT stellt zwar ein allgemein anerkanntes Konzept dar, das sich aber noch nicht in einem etablierten Versorgungsstandard niederschlägt [113, 112]. Die klinische Praxis zeigt nämlich, dass trotz Studienevidenz und Leitlinienempfehlungen die kardiale Vorlast und andere relevante hämodynamische Parameter selbst bei chirurgischen Hochrisikopatienten nur selten Bestandteil des perioperativen Monitorings sind [103]. Gemäß rezenter US-amerikanischer Studien besteht selbst bei unkomplizierten Routineeingriffen nach wie vor eine hohe Variabilität in der Menge der verabreichten Kristalloide [114, 15]. Thacker et al. [15] stellten in ihrer Studie einen signifikanten Zusammenhang zwischen hoher Flüssigkeitsgabe und der Länge des Spitalsaufenthalts sowie der Gesamtkosten fest. Darüber hinaus kam es bei Patienten mit Rektum- und Darmoperationen häufiger zu einem postoperativen Ileus. Desgleichen war eine restriktive Flüssigkeitsgabe mit einem schlechteren Outcome der Patienten verbunden. Insgesamt wurde eine 20-fache mediane Abweichung zwischen liberaler und restriktiver Flüssigkeitsgabe beobachtet.

In der Untersuchung von Lilot et al. [114] konnte die hohe Variabilität derverabreichten Kristalloidmengen nicht auf Patientenfaktoren oder Behandlungsprotokolle, sondern ausschließlich auf die Anwender und ad libitum zurückgeführt werden. In dieser Studie wurde retrospektiv die intraoperative Flüssigkeitsabgabe (2009-2011) in zwei akademischen Krankenhäusern analysiert. Ein spezifisches Flüssigkeitsprotokoll gab es nur für Prostatektomie-Patienten in einem Zentrum, wobei diese Patienten im Vergleich zu allen anderen nicht nur weniger Kristalloide verabreicht bekamen, sondern auch die Spannbreite der infundierten Kristalloidmengen deutlich geringer ausfiel, vor allem im Vergleich zu Patienten mit Appendektomien im selben Krankenhaus (korrigierter Variationskoeffizient $34 \%$ versus $97 \%$ ).

Die Autoren kommen zu dem Schluss, dass die Umsetzung von Leitlinien wie Restriktion von Kristalloiden und GDT helfen könnte, die Flüssigkeitsgabe zu standardisieren [114]. Dies wurde 2012 in einem der beiden Zentren umgesetzt und die historische Analyse vor und 
nach der Implementierung des Performance-Improvement-Projekts zeigt nicht nur eine signifikant reduzierte Flüssigkeitsverabreichung von durchschnittlich $9,9 \mathrm{ml} / \mathrm{kg} / \mathrm{h}$ auf $6,6 \mathrm{ml} / \mathrm{kg} / \mathrm{h}$ pro Fall $(p<0,01)$, sondern auch einen kürzeren Spitalaufenthalt (10 versus 7 Tage; $p=$ $0,0001)$ und signifikant weniger Komplikationen (39\% versus $25 \%$; $p=0,04$ ) [103].

Protokolle zur perioperativen hämodynamischen Stabilisierung ("goal-directed therapy", GDT) können das klinische Ergebnis der Patienten verbessern. Entsprechende Algorithmen sollten eine individualisierte Volumen- und Flüssigkeitstherapie erlauben, die auf sich ändernde physiologische Bedürfnissen und das Ansprechen auf die Flüssigkeitsgabe sowie der medikamentösen Behandlung basiert (International Fluid Optimization Group/ Camacho Navarro et al. 2015 [113]).

\section{Teil 2: HES - Einsatz \& Perspektiven in Österreich}

Die Ergebnisse der Diskussion einer Expertengruppe am 3.3.2016 in Wien über den aktuellen Einsatz von HES soll im Folgenden dargestellt werden. Die Gesprächspartner wurden nach ihren klinischen Schwerpunkten ausgewählt, sodass Expertise in den klinischen Bereichen der prähospitalen Notfallmedizin, Herzanästhesie, Intensivmedizin, Geburtshilfe, Orthopädie und großen Chirurgie verfügbar war. Die bundesweite Repräsen$\tan z$ erschien wichtig, obwohl die Verwendung von HES 2015 einheitlich war (• Abb. 1).

Die Diskussion der Experten basierte auf der Bearbeitung von klinischen Fragen, die vorab von einem Diskussionspartner erstellt wurden (SKL).

\section{Einsatz von HES bei schwerer perioperativer Blutung}

\section{Wie messen Sie die Hypovolämie?}

Die Experten sind sich einig, dass Druckparameter zum Volumenmonitoring ungeeignet sind. „Den Pulmonaliskatheter verwenden wir für etwas anderes als Volumenmonitoring, z. B. bei pulmonaler Hypertension“. „Das Outcome ist am besten, wenn die Füllungsdrücke niedrig sind.“
Die Experten stimmen darin überein, dass es derzeit noch keinen allgemein anerkannten Parameter zur intravaskulären Füllstandsmessung in der klinischen Praxis gibt. Derzeit kann eine Hypovolämie nur in Zusammenschau verschiedener Elemente abschätzend vermutet werden: dem klinischen Blick (z. B. auf den tatsächlichen Blutverlust, Rekapillarisierungszeit, Temperaturdifferenzen der Extremitäten), dem Verlauf metabolischer Parameter (z. B. gemischtvenöse Sättigung, $\mathrm{CO}_{2}$-Veränderungen), dem Vorlastparameter (z. B. mittels ösophagealer Dopplermessung, Messung der aortalen Flusszeit, Pulskonturanalyse) sowie die Visualisierung der Füllung des Herzens mittels Echokardiographie. Die Gewichtung dieser Indizien des Volumenmonitorings ist für die Experten je nach klinischem Aufgabengebiet unterschiedlich: z. B. wird im Bereich der Kinderanästhesie auf klinisches Beobachten gesetzt, mitbedingt durch fehlende Validierung und Praktikabilität einzelner apparativer Methoden. „Volumentherapie muss man am Bett machen und es braucht einen Arzt, der sich überlegt, was er tut."

In der Präklinik besteht kaum die Möglichkeit zum erweiterten hämodynamischen Monitoring, wohl aber der Bedarf der qualitativen Füllstanderfassung. Mittels Echokardiographie könnte die Ja-Nein-Frage beantwortbar sein, ob der Ventrikel weit ist oder nicht. Diagnoseunterstützung am Unfallort via Telemedizin oder soziale Medien halten die Experten in der Zukunft nicht für ausgeschlossen.

Die Anwendbarkeit von Vigileo Monitor (Edwards Lifesciences), Lidco System (LIDCO Ltd.) oder CNAP-Monitor (CNSystems) zur Vermeidung unnötiger Volumengaben wird von den Experten als gleichwertig eingestuft. Ebenso werden die Limitationen bei allen derzeitigen Systemen ähnlich wahrgenommen, z. B. bei bestehendem Vorhofflimmern.

Mikrozirkulatorische Perfusionsparameter werden derzeit von keinem Experten außer zu wissenschaftlichen Zwecken eingesetzt.

\section{Wann messen Sie Hypovolämie?}

Die Mehrheit der Experten erweitert das routinemäßige hämodynamische
Monitoring (arterieller Blutdruck, Herzfrequenz) um die oben genannten $\mathrm{Pa}$ rameter der Füllstandsmessung (abhängig vom klinischen Setting) bereits vor großen Eingriffen mit erwartetem hohem Flüssigkeitsshift, bei Risikopatienten mit eingeschränkter kardiozirkulatorischer Reserve oder bei drohender bzw. manifester Instabilität. Die Experten scheinen sich einig zu sein, dass in schwierigen klinischen Situationen, etwa bei der schweren Blutung, nicht aus dem Bauch heraus Volumentherapie gemacht werden sollte, sondern zielgerichtet basierend auf Parametern des Volumenmonitorings. Zitate hierzu: „Ohne Monitoring ist die Behandlung unsensibel.“ „Viel Erfahrung ohne Monitoringkontrolle steigert das Selbstbewusstsein, aber nicht das Können.“ „Die schwere Hypovolämie kann nicht mit Katecholaminen behandelt werden“.

Allerdings haben nur wenige Experten in ihren Abteilungen einen vordefinierten Algorithmus im Sinne einer GDT. Der Mehrwert von Protokollen beim Bewusstmachen von medizinischen Entscheidungen, als Lerneffekt für die Mitarbeiter und Faktor der Vigilanzsteigerung für Stopp-Signale zur Hypervolämievermeidung wird jedoch von allen Experten wahrgenommen.

Während auf der Intensivstation Verfahren des erweiterten hämodynamischen Volumenmonitorings liberal eingesetzt werden, erfolgt dies in der perioperativen Intensivmedizin im Operationssaal restriktiv. Laut Experten könnte die Hemmschwelle für die Pulskonturanalyse in manchen Patientengruppen (z. B. ohne andere Indikation für eine arterielle Punktion) durch den nichtinvasiven CNAP abgebaut werden, wenn nämlich nur der Fingersensor aufgesetzt, aber keine Arterienkanüle gestochen werden muss. Die Notwendigkeit von klinischen Studien und Anwendungsbeobachtungen wird einhellig gefordert.

\section{Geben Sie HES bei Hypopvolämie?}

Bei Hypovolämie besteht eine klare Indikation für HES: Die Mehrzahl der Experten infundiert HES bei vermuteter bzw. gemessener Hypovolämie, insbesondere im prähospitalen Setting, im Schockraum, bei schwerer Blutung un- 
geachtet des Ortes des Auftretens, bei kindlichen und geriatrischen Patienten, in der Transplantationsmedizin und bei peripartaler Blutung. Die Infusionsdosis wird stets zurückhaltend gewählt (Boli zu 100-250 ml). Die Kontraindikationen mit bestehender oder vermuteter Nieren- und Leberfunktionsstörung werden von den Experten sehr ernst genommen; Humanalbumin wird in dieser klinischen Situation bzw. bei gleichzeitig bestehender Hypalbuminämie mit und ohne Aszites bevorzugt.

Bei dieser klinischen Frage wird erkennbar, dass sämtliche Experten die hämodynamische Stabilisierung primär mit Kristalloiden in eskalierenden Dosen austesten - trotz theoretischen Wissens über deren niedrige Volumenwirksamkeit. Erst bei therapierefraktärer Hypovolämie bzw. bei bereits bestehenden Ödemen wird HES verordnet. Hinter dieser Indikationshierarchie stehen medikolegale Überlegungen, weil ein derartiges Vorgehen im Zulassungstext empfohlen wird sowie die Sorge um Nebenwirkungen von HES, insbesondere auf die Nierenfunktion. Auch wenn für einige Experten die Daten und Metaanalysen seit 1999 diesbezüglich nicht aussagekräftig sind, weil in den Primärstudien selbst kein adäquates Hypovolämie-Monitoring als Indikation und Dosisanleitung für HES eingesetzt wurden und höchstwahrscheinlich eine Überdosierung von HES erfolgt ist, so bleibt doch für alle Experten zumindest ein Rest an Sicherheitsbedenken, selbst bei indikationskonformer Anwendung von HES. Im Gegensatz zu diesen vermeintlich evidenzbasierten Befürchtungen berichten die Experten von der wiederholten Beobachtung, dass HES nierenprotektiv wirkt, wenn es frühzeitig und kurzfristig zur hämodynamischen Stabilisierung gegeben wird.

Anstatt HES wird bei Hypovolämie anwenderabhängig auch Gelatine oder Albumin eingesetzt, obwohl es weniger Daten sowohl zur Wirksamkeit als auch zur Sicherheit gibt. Es fällt in der Diskussion auf, dass mit reinem blutdruckund herzfrequenzbasiertem Monitoring die gegebene Dosis dieser Komparatoren scheinbar gleich groß ist. Aussagen über Volumenwirksamkeit und die Kontext- sensitivität (Infusion hat bei Hypovolämie den höchsten Wirkungsgrad) setzen also ein spezifisches Volumenmonitoring voraus.

Im klinischen Alltag werden im OP überwiegend nur Kristalloide restriktiv verabreicht, weil die Mehrzahl der Patienten nicht hypovolämisch ist. Alle Experten bevorzugen im OP und präklinisch HES als Kolloid, auf der Intensivstation Gelatine und Albumin.

\section{Wie messen Sie Koagulopathie?}

In Österreich hat sich die Rotationsthromboelastometrie (ROTEM, TEM International) auf den meisten Anästhesieabteilungen durchgesetzt. Die Messung der Thrombozytenfunktion und der Anti-Xa-Spiegel wird situationsangepasst eingesetzt

\section{Wie behandeln Sie die Koagulopathie?}

In den letzten Jahrzehnten ist man vom blinden Einfüllen von FFP weggegangen hin $\mathrm{zu}$ einer ROTEM-zielgerichteten Therapie bei Erwachsenen mit Antifibrinolytika, Thrombozytenkonzentraten und/oder Gerinnungsfaktorenkonzentraten, allen voran Fibrinogenkonzentrat bei Hypofibrinogenämie. „Wir behandeln die erworbene Koagulopathie in Österreich quasi FFP-frei." Alle Experten lehnen FFP auch zur reinen Hypovolämiekorrektur ab. Nicht einmal der etwaige Anstieg des kolloidosmotischen Drucks durch FFP wird als wichtig erachtet, auch nicht bei Lungeneingriffen. Der Nebeneffekt der Zufuhr von Volumen durch FFP wird jedoch als günstig erachtet bei einer mittlerweile selten notwenigen Massivtransfusion.

Der Weg zum potenten Therapeutikum bei entsprechender Indikation ist im Blutungsmanagement mittlerweile evidenzbasiert und leitlinienkonform [99]. Übrigens sehen einige Experten die Entwicklungen im Bereich des Volumenmanagements (ähnlich zum Blutungsmanagement): hin zum potenten Medikament (Kolloid) bei durch Messen quantifiziertem Bedarf und der nachgewiesenen Indikation. „Geben, wenn's fehlt“.

\section{Geben Sie HES bei Koagulopathie?}

Die Mehrheit der Experten therapiert einen Volumenmangel mit HES (volu- menmonitoringgezielt und restriktiv dosiert) selbst bei gleichzeitig bestehender koagulopathischer Blutung während gezielter Gerinnungstherapie. „Wir haben gelernt, dass der vorsichtige Ersatz von HES sogar zu weniger Blutung führt.“

\section{Veranlassen Sie die Kontrolle der Nierenfunktionstestung 90 Tage nach HES-Gabe?}

Keiner der Experten veranlasst diese Messung, weil es nicht sinnvoll und nicht durchführbar erscheint. Man kann daraus in der perioperativen Medizin mit Anwendung unterschiedlicher Medikamente mit potenziellen Nebenwirkungen auf die Nierenfunktion (z. B. nicht steroidale Antirheumatika, Antibiotika) nichts ablesen. Aus medikolegalen Gründen kann es überlegenswert sein, die Kreatinin-Clearance zu kontrollieren, wenn der Patient so lange im Spital sein sollte.

\section{Einsatz von HES in der Präklinik und im Schockraum}

\section{Geben Sie HES beim Polytrauma- verletzten?}

Sowohl in der Präklinik als auch im Schockraum hat HES als einziges Kolloid einen fixen Stellenwert. Experten, die derzeit nicht in diesem klinischen Setting arbeiten, würden auch überwiegend HES bei einer Traumablutung geben.

\section{Haben Sie eine Obergrenze von HES beim Polytraumaverletzten?}

Die Dosisobergrenze von HES orientiert sich in der Präklinik am Zeitfaktor. In der Regel werden nicht mehr als 0,5-1 1 verabreicht. Eine $250 \mathrm{ml}$ Bolusgröße als Interventionseinheit erscheint sinnvoll. Die zugelassene Tagesmaximaldosis von $30 \mathrm{ml} / \mathrm{kg}$ HES sollte nicht überschritten werden, auch wenn es durch die noch unkontrollierte Blutungsquelle aus dem Verletzten herausrinnt. Bei Massivblutung würden alle Experten bis zum ersten Blutungsstopp primär allogene Blutprodukte geben (also Erythrozytenkonzentrate und Frischplasma) und die autologe Blutaufbereitung nutzen, aber es sollte sobald wie möglich auf die gezielte Gabe von Kolloid und Gerinnungsfaktorenkonzentraten umgestiegen werden. 
Permissive Hypotension mit einem systolischen Blutdruck von 80 bis $100 \mathrm{~mm} \mathrm{Hg}$ ist gelebte Praxis. Insbesondere bei Polytraumen sollte dieser Zielblutdruck vor definitiver Blutstillung nicht überschritten werden (Ausnahme Schädel-Hirn-Trauma).

\section{Einsatz von HES in der Herzanästhesie}

\section{Geben Sie HES in die Herzlungen- maschine?}

„Ja, weil es die Chirurgen so wollen.“ Ein Experte verwendet HES auch in der Kinderherzanästhesie. Experten, die derzeit nicht aufder Herzanästhesie arbeiten, würden HES oder Gelatine zum Pump Prime einsetzen.

\section{Geben Sie HES bei Hypovolämie \\ i. v.?}

Ein Experte spricht sich klar dagegen aus, weil eigene Studien das Signal der Erhöhung der Transfusionsrate ohne irgendeinen klinischen Benefit zeigten. HES wurde hierbei nicht nach GDT gesteuert.
Einsatz von HES in der Geburtshilfe

\section{Geben Sie HES bei der schweren peripartalen Blutung?}

Diese potenziell lebensbedrohliche Situation ist eine klassische Indikation für Kolloide wie HES und Gelatine [115].

\section{Geben Sie HES zur Vorbeugung oder Behandlung von Blutdruckabfällen bei der Spinalanästhesie?}

Die österreichischen Experten wählen in diesem klinischen Setting selten HES als Pre- oder Co-Loading, weil als Ursache der nicht blutungsbedingten hämodynamischen Instabilität nur ein distributives Problem vorliegt. Wenn eine Blutdruckstabilisierung der Mutter erforderlich erscheint, wird zumeist ein Kristalloid in Kombination mit Phenylephrin oder Ephedrin gegeben. Katecholamine wirken bei Sympathikolyse/Vasoplegie und der zuvor normovolämen/hypervolämen Gebärenden schneller als ein Kolloidbolus. Es wird jedoch auch auf mögliche Nebenwirkungen durch pharmakolo- gisch hervorgerufene Vasokonstriktion, wie verminderte Plazentaperfusion hingewiesen. Konventionell wird der systolische maternale Blutdruck über $100 \mathrm{~mm} \mathrm{Hg}$ erhalten; wichtiger als der Volumenstatus der Mutter erscheinen jedoch die Blutgasanalyse aus der $\mathrm{Na}$ belschnur und die Vitalität des Kindes.

\section{Einsatz von HES auf der Intensivstation}

\section{Wie oft sehen Sie schwere Blutungen auf der Intensivstation?} Relevante Blutungen sind selten und erfordern in der Beobachtung der Experten meist eine chirurgische Revision. Die Rate an solchen schweren Blutungen spiegelt sich also an den sehr geringen OPabhängigen Revisionsraten wider.

\section{Geben Sie HES bei schwerer postoperativer Blutung?}

Aus medikolegalen Gründen verwendet keiner der Experten HES auf der Intensivstation, außer es blutet stark. Seit der Empfehlung der Zulassungsbehörde wer-

Hier steht eine Anzeige.

\section{Springer}


den auf Intensivstationen bei Hypovolämie Gelatine oder Albumin als Kolloide verwendet. Die Ergebnisse der in Teil 1 zusammengefassten großen Studien werden von allen Experten infrage gestellt; es gibt also keine evidenzbasierten Gründe für die HES-Abstinenz in den Räumlichkeiten einer Intensivstation. 2015 wurden 7 anaphylaktische Fälle durch Gelatine gemeldet. Das anaphylaktische Potenzial der Gelatinelösungen und der geringere Volumeneffekt veranlassen manche Experten zur Bevorzugung von Albumin auf der Intensivstation.

Über die Notwendigkeit der Vermeidung von allogenen Blutprodukten auch auf der Intensivstation sind sich die Experten einig. „Es ist nicht egal, wie viele Liter an Transfusionen hineinrinnen."

Die Experten verwenden keine Scores oder Formeln zur Berechnung eines vermeintlichen Volumenbedarfs, sondern indizieren und dosieren nur nach individuell und bettseitig erfasstem Bedarf. Den Grundsatz der restriktiven Volumengabe erachten die Experten auch im Intensivsetting als essenziell. „Patienten kämpfen lange, um das überschüssige Volumen wieder los zu werden." Obwohl Kristalloide rascher zu Ödemen führen, dürfte das Ausschwemmen aus dem Organismus beim Genesungsprozess schneller sein als bei Kolloiden.

Die Bewertung der Experten hinsichtlich der Ablagerungen der HES-Moleküle im Körper ist uneinheitlich: Manche stufen die rasche Schockstabilisierung und Vermeidung von Folgeschäden als vorrangig ein und nehmen eine $\mathrm{Ab}$ lagerung von Molekülen dafür in Kauf. Andere haben Sorge wegen dieser Langzeitablagerung: „Das intrazelluläre Speichern macht mich nervös." Allerdings gibt es zu bedenken: „Es gibt nichts, woraus hervorgeht, dass die Ablagerung im Körper zu klinischen Funktionseinschränkungen führt.“

\section{"Patient blood management" (PBM)}

\section{Verwenden Sie ein Kolloid zur Steigerung der Anämietoleranz?} Nur zwei Experten setzen dazu gezielt HES ein. Andere Experten verneinen dies, weil PBM noch nicht implementiert wurde.

\section{Ausbildung}

\section{Gibt es genug Möglichkeiten der Aus- und Weiterbildung?}

Zum komplexen Thema der Volumentherapie gibt es laut Experten genügend Fortbildungsveranstaltungen mit Frontalvorträgen. Es besteht jedoch ein Manko im Basiswissen zur Physiologie der Makround Mikrozirkulation sowie der Methodologie von Verfahren des Volumenmonitorings. Interaktives e-learning, Lernen an Fällen mit aktiver Fragenbeantwortung, Simulationstraining zum erweiterten hämodynamischen Monitoring und Volumentherapie könnten helfen.

Kritisiert wird von den Experten, dass Vorträge im Bereich der Flüssigkeitstherapie mit und ohne Firmensponsoring rasch ins Ideologische und Philosophische abrutschen. Es braucht mehr unbeeinflusste Lehre durch Fachgesellschaften.

\section{Korrespondenzadresse}

Univ.-Prof. Dr. S. Kozek-Langenecker, MBA

Evangelisches Krankenhaus Wien

Hans-Sachs-Gasse 10-12, 1180 Wien,

Österreich

sibylle.kozek@aon.at

Danksagung. Die Manuskripterstellung erfolgte in Zusammenarbeit mit Dr. Monika Peretz, Update Europe, Gesellschaft für ärztliche Fortbildung.

Open access funding provided by Medical University of Vienna.

\section{Einhaltung ethischer Richtlinien}

Interessenkonflikt. W. Fartek, G. Gemes, M. Hiesmayr, R. Likar, G. Luckner, W. Mitterndorfer und C. Torgersen geben an, dass kein Interessenkonflikt besteht. B. Kabon erhielt Forschungsunterstützung von FreseniusKabi. S. Kozek-Langenecker erhielt Vortragshonorare von Fresenius-Kabi, B. Braun.

Dieser Beitrag beinhaltet keine von den Autoren durchgeführten Studien an Menschen oder Tieren.

Open Access. Dieser Artikel wird unter der Creative Commons Namensnennung 4.0 International Lizenz (http://creativecommons.org/licenses/by/4.0/deed. de) veröffentlicht, welche die Nutzung, Vervielfältigung, Bearbeitung, Verbreitung und Wiedergabe in jeglichem Medium und Format erlaubt, sofern
Sie den/die ursprünglichen Autor(en) und die Quelle ordnungsgemäßnennen, einen Link zur Creative Commons Lizenz beifügen und angeben, ob Änderungen vorgenommen wurden.

\section{Literatur}

1. Marx G (2015) Neue AWMF-S3-Leitlinie Volumentherapie. Auf Wiedersehen Emotionen willkommen Evidenz. Med Klin Intensivmed Notfmed 110:108-109

2. Hogan JJ (1915) The intravenous use of colloidal (gelatin) solutions in shock. J Am Med Assoc 64:721-726

3. Böhm R, Meybohm P (2011) Volumenersatztherapie. Notfmed Up2 date 6:2-4

4. Bundgaard-Nielsen $M$, Secher $N H$, Kehlet $H$ (2009) „Liberal" vs. „restrictive" perioperative fluid therapy - a critical assessment of the evidence. Acta Anaesthesiol Scand 53:843-851

5. Dubin A, Pozo MO, Casabella CA et al (2010) Comparison of $6 \%$ hydroxyethyl starch $130 / 0.4$ and saline solution for resuscitation of the microcirculation during the early goal-directed therapy of septic patients. J Crit Care 25:659:e1-e8

6. Jacob M, Chappell D, Hofmann-Kiefer Ket al (2012) The intravascular volume effect of Ringer's lactate is below $20 \%$ : a prospective study in humans. Crit Care 16:R86

7. Jacob M, Chappell D (2009) Mythen und Fakten der perioperativen Infusionstherapie. Anaesthesiol Intensivmed 6:358-376

8. Adams HA (2007) Volumen- und Flüssigkeitsersatz - Physiologie, Pathophysiologie, Pharmakologie und klinischer Einsatz (Teil I). Anasthesiol Intensivmed 48:448-460

9. Balogh Z, McKinley BA, Cocanour CS et al (2003) Supra-normal trauma resuscitation causes more cases of abdominal compartment syndrome. Arch Surg 138:637-643

10. Maxwell RA (1999) Secondary abdominal compartment syndrome: an underappreciated manifestation of severe hemorrhagic shock. J Trauma 47:995-999

11. Boyd JH, Forbes J, Nakada TA et al (2011) Fluid resuscitation in septic shock: a positive fluid balance and elevated central venous pressure are associated with increased mortality. Crit Care Med 39(2):259-265

12. Payen D, de Pont AC, Sakr Y, et al, Sepsis Occurrence in Acutely III Patients (SOAP) Investigators (2008) A positive fluid balance is associated with a worse outcome in patients with acute renal failure. Crit Care 12(3):R74

13. Hamilton MA, Mythen MG, Ackland GL (2006) Less is not more: a lack of evidence for intraoperative fluid restriction improving outcome after major elective gastrointestinal surgery. Anesth Analg 102:970-971

14. Holte K, Foss NB, Andersen J et al (2007) Liberal or restrictive fluid administration in fast-track colonic surgery: a randomized, double-blind study. $\mathrm{Br}$ J Anaesth 99:500-508

15. Thacker JK, Mountford WK, Ernst FR et al (2016) Perioperative fluid utilization variability and association with outcomes: considerations for enhanced recovery efforts in sample US surgical populations. Ann Surg 263(3):502-510

16. Westphal M, James MF, Kozek-Langenecker $S$ et al (2009) Hydroxyethyl starches: different products different effects. Anesthesiology 111(1):187-202

17. Deusch E, Gamsjäger T, Kress HG, Kozek-Langenecker S (2003) Binding of hydroxyethyl starch 
molecules to the platelet surface. Anesth Analg 97(3):680-683

18. Kozek-Langenecker SA (2015) Fluids and coagulation. Curr Opin Crit Care 21(4):285-291

19. Hartl WH, Rittler P (2002) Perioperative Infusionstherapie-Prinzipien. Chirurg 73:1067-1086

20. Kozek-Langenecker SA (2005) Effects of hydroxyethyl starch solutions on hemostasis. Anesthesiology 103(3):654-660

21. Rehm M, Orth VH, Kreimeier U et al (2001) Changes in blood volume during acute normovolemic hemodilution with $5 \%$ albumin or $6 \%$ hydroxyethyl starch and intraoperative retransfusion. Anaesthesist 50:569-579

22. Barron ME, Wilkes MM, Narvickis RJ (2004) A systematic review of the comparative safety of colloids. Arch Surg 193:552-563

23. Laxenaire MC, Charpentier C, Feldmann L (1994) Réactions anaphylactoides aux substituts colloidaux du plasma: incidence de risque, mécanismes. Ann Fr Anesth Reanim 13:301-310

24. de Jonge $E$, Levi $M$, Berends $F$, van der Ende $A E$, ten Cate JW, Stoutenbeek CP (1998) Impaired haemostasis by intravenous administration of a gelatin-based plasma expander in human subjects. Thromb Haemost 79(2):286-290

25. Engvall E, Ruoslahti E, Miller EJ (1978) Affinity of fibronectin to collagens of different genetic types and to fibrinogen. J Exp Med 147(6):1584-1595

26. Mardel SN, Saunders FM, Allen H et al (1998) Reduced quality of clot formation with gelatin-based plasma substitutes. Br J Anaesth 80(2):204-207

27. Thaler U, Deusch E, Kozek-Langenecker SA (2005) In vitro effects of gelatin solutions on platelet function: a comparison with hydroxyethyl starch solutions. Anaesthesia 60(6):554-559

28. Moerman A, van Eeckhout C, Vanderstraeten $K$, de Somer F, van Belleghem Y, de HertS (2016) he effect of hydroxyethyl starch $6 \%$ 130/0.4 compared with gelatin on microvascular reactivity. Anaesthesia 71:798. doi:10.1111/anae.13388

29. ÖztürkT, OnurE, Cerrahoğlu M, Çalgan M, Nizamoglu F, Çivi M (2015) Immune and inflammatory role of hydroxyethyl starch 130/0.4 and fluid gelatin in patients undergoing coronary surgery. Cytokine 74(1):69-67

30. Dietrich H-J (2001) Kristalloide versus Kolloide A never ending story? Anaesthesist 50:432-435

31. Rehm M (2013) Anwendungsbeschränkung für Hydroxyäthylstärke - Hintergründe und alternative Konzepte. Anaesthesist 62:644-655

32. Moeller C, Fleischmann C, Thomas-Rueddel D et al (2016) How safe is gelatin? A systematic review and metaanalysis of gelatin-containing plasma expanders versus crystalloids and albumin. J Crit Care 35:75. doi:10.1016/j.jcrc.2016.04.011

33. Rehm M (2013) Hydroxyäthylstärke. Anaesthesist 62:926-930 (Erwiderung aufLeserbrief von Adams HA \& Fries D)

34. [FDA 1978+2015]. Department of Health and Human Services, Food and Drug Administration 21 CFR Part 216 [Docket No. 98 N-0655] List of Drug Products That Have Been Withdrawn or Removed From the Market for Reasons of Safety or Effectiveness (see the Federal Register of April 7, 1978 (43 FR 14743)). In: FDA Briefing Document. Pharmacy Compounding Advisory Committee (PCAC) Meeting. February 23-24, 2015. URL: http:// www.fda.gov/downloads/AdvisoryCommittees/ CommitteesMeetingMaterials/Drugs/ PharmacyCompoundingAdvisoryCommittee/ UCM433804.pdf.Zugegriffen:6. Mai 2016

35. BurdettE, Dushianthan A, Bennett-Guerrero E et a (2012) Perioperative buffered versus non-buffered fluid administration for surgery in adults. Cochrane Database Syst Rev 12:CD004089. doi:10.1002/ 14651858.cd004089.pub2

36. Roquilly A, Loutrel O, Cinotti Ret al (2013) Balanced versus chloride-rich solutionsforfluid resuscitation in brain-injured patients: a randomised doubleblind pilot study. Crit Care 17(2):R77

37. Yunos NM, Bellomo R, Hegarty C et al (2012) Association between a chloride-liberal vs chloriderestrictive intravenous fluid administration strategy and kidney injury in critically ill adults. JAMA 308(15):1566-1572

38. Young P, Bailey M, Beasley R, et al, SPLIT Investigators, ANZICS CTG (2015) Effect of a Buffered Crystalloid Solution vs Saline on Acute Kidney Injury Among Patients in the Intensive Care Unit: The SPLIT Randomized Clinical Trial. JAMA 314(16):1701-1710

39. Kellum JA, Shaw AD (2015) Assessing toxicity of intravenous crystalloids im critically ill patients. JAMA 314(16):1695-1696

40. Roessler M, Bode K, Bauer M (2014) Volumentherapie bei Hämorrhagie. Anaesthesist 63:730-744

41. Jenssen T, Nurjhan N, Consoli A, Gerich JE (1993) Dose-response effects of lactate infusions on gluconeogenesis from lactate in normal man. Eur JClin Invest 23(8):448-454

42. Chertoff J, Chisum M, Simmons L, King B, Walke M, Lascano J (2016) Prognostic utility of plasma lactate measured between 24 and $48 \mathrm{~h}$ after initiation of early goal-directed therapy in the management of sepsis, severe sepsis, and septic shock. J Intensive Care 4:13

43. Levraut J, Ciebiera JP, Chave $S$ et al (1998) Mild hyperlactatemia in stable septic patients is due to impaired lactate clearance rather than overproduction. Am J Respir Crit Care Med 157:1021-1026

44. LevrautJ,IchaiC, Petitletal(2003) Lowexogeneous lactate clearance as an early predictor of mortality in normolactemic critically ill septic patients. Crit Care Med 31:705-710

45. Striebel HW (2014) Band I Grundlagen - Formen der Allgemeinanästhesie - Lokal- und Regionalanästhesie - Besonderheiten - Narkoseprobleme Band II Nebenerkrankungen - Fachspezifische Anästhesie - Aufwachraum - Lebensrettende Sofortmaßnahmen - Anhang, 3. Aufl. Schattauer, Stuttgart

46. Jacob M, Rehm M, Orth V et al (2003) Exact measurement of the volume effect of $6 \%$ hydoxyethyl starch 130/0.4 (Voluven) during acute preoperative normovolemic hemodilution. Anaesthesist 52:896-904

47. Rehm M, Orth V, Kreimeier U et al (2000) Changes in intravascular volume during acute normovolemic hemodilution and intraoperative retransfusion in patients with radical hysterectomy. Anesthesiology 92:657-664

48. Chappell D, Jacob M, Hofmann-Kiefer K, Conzen P, Rehm M (2008) A rational approach to perioperative fluid management. Anesthesiology 109:723-740

49. Hiltebrand LB, Kimberger O, Arnberger $M$ et al (2009) Crystalloids versus colloids for goaldirected fluid therapy in major surgery. Crit Care 13:R40

50. Kimberger O, Arnberger M, Brandt S et al (2009) Goal-directed colloid administration improves the microcirculation of healthy and perianastomotic colon. Anesthesiology 110:496-504

51. Allison KP, Gosling P, Jones $S$ et al (1999) Randomized trial of hydroxyethyl starch versus gelatine for trauma resuscitation. J Trauma 47:1114-1121
52. Lobo SM, Orrico SR, Queiroz MM et al (2008) Comparison of the effects of lactated Ringer solution with and without hydroxyethyl starch fluid resuscitation on gut edema during severe splanchnic ischemia. Braz J Med Biol Res 41:634-639

53. Marx G, PedderS, Smith Letal (2006) Attenuation of capillaryleakage by hydroxyethylstarch (130/0.42) in a porcine model of septic shock. Crit Care Med 34:3005-3010

54. Mcllroy DR, Kharasch ED (2003) Acute intravascular volume expansion with rapidly administered crystalloid or colloid in the setting of moderate hypovolemia. Anesth Analg 96(6):1572-1577

55. Prien T, Backhaus N, Pelster F et al (1990) Effect of intraoperative fluid administration and colloid osmotic pressure on the formation of intestinal edema during gastrointestinal surgery. J Clin Anesth 2:317-323

56. Schramko A, Suojaranta-Ylinen R, Kuitunen A et al (2010) Hydroxyethylstarch and gelatin solutions impair blood coagulation after cardiac surgery: a prospective randomized trial. $\mathrm{Br} \mathrm{J}$ Anaesth 104:691-697

57. Zdolsek JH, Bergek C, Lindahl TL, Hahn RG (2015) Colloid osmotic pressure and extravasation of plasma proteins following infusion of Ringer's acetate and hydroxyethyl starch 130/0.4. Acta Anaesthesiol Scand 59(10):1303-1310

58. Roger C, Muller L, Deras P et al (2014) Does the type of fluid affect rapidity of shock reversal in an anaesthetized-piglet model of near-fatal controlled haemorrhage? A randomized study. $\mathrm{Br}$ JAnaesth 112(6):1015-1023

59. Müller RB, Ostrowski SR, Haase N et al (2016) Markers of endothelial damage and coagulation impairment in patients with severe sepsis resuscitated with hydroxyethyl starch $130 / 0.42$ vs Ringer acetate. J Crit Care 32:16-20

60. Becker BF, Chappell D, Jacob M (2010) Endothelial glycocalyxand coronary vascular permeability: the fringe benefit. Basic Res Cardiol 105(6):687-701

61. Chappell D, Westphal M, Jacob M (2009) The impact of the glycocalyx on microcirculatory oxygen distribution in critical illness. Curr Opin Anaesthesiol 22:155-162

62. Jacob M, Bruegger D, Rehm M et al (2007) The endothelial glycocalyx affords compatibility of Starling's principle and high cardiac interstitial albumin levels. Cardiovasc Res 2007(73):575-586

63. Rehm M, Zahler S, Lotsch Metal (2004) Endothelial glycocalyx as an additional barrier determining extravasation of $6 \%$ hydroxyethyl starch or $5 \%$ albumin solutions in the coronary vascular bed. Anesthesiology 100:1211-1223

64. Chappell D, Jacob M (2012) Die endotheliale Glykokalyx: Ein neues Target der Flüssigkeitstherapie. Intensiv News 16(1-2):1-4

65. Chappell D, Hofmann-Kiefer K, Jacob M et al (2009) TNF-alpha induced shedding of the endothelia glycocalyx is prevented by hydrocortisone and antithrombin. Basic Res Cardiol 104:78-89

66. Rehm M, Bruegger D, Christ F et al (2007) Shedding of the endothelial glycocalyx in patients undergoing major vascular surgery with global and regional ischemia. Circulation 116:1896-1906

67. Chappell D, Bruegger D, Potzel J et al (2014) Hypervolemia increases release of atrial natriuretic peptide and shedding of the endothelial glycocalyx. Crit Care 18(5):538

68. Chappell D, Jacob M (2013) Hydroxyethyl starch the importance of being earnest. Scand J Trauma Resusc Emerg Med 21:61

69. Gillies MA, Habicher M, Jhanji S et al (2014) Incidence of postoperative death and acute kidney injury associated with i. v. $6 \%$ hydroxyethyl starch 
use: systematic review and meta-analysis. $\mathrm{Br}$ J Anaesth 112:25-34

70. Hamilton MA, Cecconi M, Rhodes A (2011) A systematic review and metaanalysis on the use of preemptive hemodynamic intervention to improve postoperative outcomes in moderate and high-risk surgical patients. Anesth Analg 112:1392-1402

71. Mahmood A, Gosling P, Vohra RK (2007) Randomized clinical trial comparing the effects on renal function of hydroxyethyl starch or gelatine during aortic aneurysm surgery. Br JSurg 94(4):427-433

72. Martin C, Jacob $M$, Vicaut $E$, Guidet $B$, van Aken H, Kurz A (2013) Effect of waxy maize-derived hydroxyethyl starch 130/0.4 on renal function in surgical patients. Anesthesiology 118:387-394

73. van der Linden $P$, James $M$, Mythen $M$, Weiskopf RB (2013) Safety of modern starches used during surgery. Anesth Analg 116:35-48

74. Brunkhorst FM, Engel Ch, Bloos F et al (2008) Intensive insulin therapy and pentastarch resuscitation in severe sepsis. NEngl J Med 358:125-139

75. Perner A, Haase N, Guttormsen AB et al (2012) Hydroxyethylstarch 130/0.42 versus Ringer's acetate in severe sepsis. N Engl J Med 367:124-134

76. Myburgh JA, Finfer S, Bellomo R, et al, CHEST Investigators, Australian and New Zealand Intensive Care Society Clinical Trials Group (2012) Hydroxyethyl starch or saline for fluid resuscitation in intensive care. N Engl J Med 367(20):1901-1911

77. PRAC (2013) PRAC confirms that hydroxyethylstarch solutions (HES) should no longer be used in patients with sepsis or burn injuries or in critically ill patients. http://www.ema.europa.eu/docs/en GB/document_library/Press_release/2013/10/ WC500151964.pdf.Zugegriffen: 10. Feb. 2016

78. EMA (2013) Hydroxyethylstärke(HES)-Lösungen dürfen bei Patienten mit Sepsis, mit Verbrennungen und bei intensivpflichtigen Patienten nicht mehr angewendet werden. http://www. ema.europa.eu/docs/de_DE/document_library/ Referrals document/Solutions for infusion containing_hydroxyethyl_starch/European_ Commission_final_decision/WC500162361.pdf. Zugegriffen: 10. Feb. 2016

79. Schortgen F, Lacherade JC, Bruneel F, Cattaneo I, Hemery F, Lemaire F, Brochard L (2001) Effects of hydroxyethylstarch and gelatin on renal function in severe sepsis: a multicentre randomised study. Lancet 357:911-916

80. Dellinger RP, Levy MM, Rhodes A et al (2013) Surviving Sepsis Campaign Guidelines Committee including The Pediatric Subgroup. Surviving Sepsis Campaign: international guidelines for management of severe sepsis and septic shock, 2012. Intensive Care Med 39(2):165-228

81. Guidet B, Martinet O, Boulain T et al (2012) Assessment of hemodynamic efficacy and safety of $6 \%$ hydroxyethylstarch $130 / 0.4$ vs. $0.9 \% \mathrm{NaCl}$ fluid replacement in patients with severe sepsis: the CRYSTMAS study. Crit Care 16:R94

82. Rivers E, Nguyen B, Havstad S et al (2001) Early Goal-Directed Therapy Collaborative G: Early goaldirected therapy in the treatment of severe sepsis and septicshock. NEnglJMed 45:1368-1377

83. Annane D, Siami S, Jaber S et al (2013) CRISTAL Investigators. Effects of fluid resuscitation with colloids vs crystalloids on mortality in critically ill patients presenting with hypovolemic shock: the CRISTAL randomized trial. JAMA 310:1809-1817

84. Deutsche Gesellschaft für Anästhesiologie und Intensivmedizin (2014) S3-Leitlinie Intravasale Volumentherapie beim Erwachsenen. Hompage der Arbeitsgemeinschaft Wissenschaftlich Me- dizinischer Fachgesellschaften, Registernummer 001-020. http://www.awmf.org/leitlinien/detail/ II/001-020.html.Zugegriffen: 10. Feb. 2016

85. Weyland A, Scheeren T (2012) Hämodynamisches Monitoring - Verbessertes Outcome durch erweitertes perioperatives hämodynamisches Monitoring. Anasthesiol Intensivmed Notfallmed Schmerzther 47:92-100

86. Kumar A, Anel R, Bunnell E et al (2004) Pulmonary artery occlusion pressure and central venous pressure fail to predict ventricular filling volume, cardiac performance, or the response to volume infusion in normal subjects. Crit Care Med 32:691-699

87. Marik PE, Baram M, Vahid B (2008) Does central venous pressure predict fluid responsiveness? A systematic review of the literature and the tale of seven mares. Chest 134:172-178

88. Marik PE, Cavallazzi R, Vasu T, Hirani A (2009) Dynamic changes in arterial waveform derived variables and fluid responsiveness in mechanically ventilated patients: a systematic review of the literature. Crit Care Med 37:2642-2647

89. Kozek-Langenecker SA, Afshari A, Albaladejo $P$ et al (2013) Management of severe perioperative bleeding: guidelines from the European Society of Anaesthesiology. Eur J Anaesthesiol 2013(30):270-382

90. Berkenstadt H, Margalit N, Hadani M et al (2001) Stroke volume variation as a predictor of fluid responsiveness in patients undergoing brain surgery. Anesth Analg 92:984-989

91. Marik PE, Cavallazzi R (2013) Does the central venous pressure predict fluid responsiveness? An updated meta-analysis and a plea for some common sense. Crit Care Med 41:1774-178

92. Michard F, Boussat S, Chemla D et al (2000) Relation between respiratory changes in arterial pulse pressure and fluid responsiveness in septic patients with acute circulatory failure. Am J Respir Crit Care Med 162:134-138

93. Michard F, Teboul JL (2001) Predicting fluid responsiveness in ICU patients. A critical analysis of the evidence. Chest 121:2000-2008

94. Benes J, Giglio M, Brienza N, Michard F (2014) The effects of goal-directed fluid therapy based on dynamic parameters on post-surgical outcome: a meta-analysis of randomized controlled trials. Crit Care 18(5):584

95. Wagner JY, Saugel B (2015) When should we adopt continuous noninvasive hemodynamic monitoring technologies into clinical routine? JClin Monit Comput 29:1-3

96. Benes J, Haidingerova L, Pouska J et al (2015) Fluid management guided by a continuous non invasive arterial pressure device is associated with decreased postoperative morbidity after total knee and hip replacement. BMC Anesthesiol 15:148

97. Biais M, Stecken L, Ottolenghi L et al (2011) The ability of pulse pressure variations obtained with CNAP $^{T M}$ device to predict fluid responsiveness in the operating room. Anesth Analg 113:523-528

98. Adams HA, Baumann G, Cascorbi I et al (2005) Zur Diagnostik und Therapie der Schockformen. Empfehlungen der interdisziplinären Arbeitsgruppe Schock der DIVI - Teil I. Anasthesiol Intensivmed 46:63-69

99. Kozek-Langenecker SA, Bettelheim P, Giurea A et al (2013) Interdisziplinäre Empfehlung zum Anämiemanagement (Patienten-orientiertes Blutmanagement).Version 1.1 (www.oegari.at)

100. Manski D (2015) Urologielehrbuch.de. Dr. Dirk Manski, Augsburg
101. Habler $O$ (2012) Erhöhung und Ausschöpfung der natürlichen Anämietoleranz. Patient Blood Management, 9. Teil. Klinik 1:35-38

102. Benes J, Chytra I, Altmann P et al (2010) Intraoperative fluid optimization using stroke volume variation in high risk surgical patients: results of prospective randomized study. Crit Care 14:R118

103. Cannesson M, Ramsingh D, Rinehart J et al (2015) Perioperative goal-directed therapy and postoperative outcomes in patients undergoing highrisk abdominal surgery: a historical-prospective, comparative effectiveness study. Crit Care 19:261

104. Cecconi M, Corredor C, Arulkumaran N et al (2013) Clinical review: Goal-directed therapy - what is the evidence in surgical patients? The effect on different riskgroups. Crit Care 17:20

105. Donati A, Loggi S, Preiser JC et al (2007) Goaldirected intraoperative therapy reduces morbidity and length of hospital stay in high-risk surgical patients. Chest 132:1817-1824

106. Goepfert MS, Richter HP, zu Eulenburg Cetal (2013) Individually optimized hemodynamic therapy reduces complications and length of stay in the intensive care unit: a prospective, randomized controlled trial. Anesthesiology 119:824-836

107. Arulkumaran N, Corredor C, Hamilton MA et al (2014) Cardiac complications associated with goaldirected therapy in high-risk surgical patients: a meta-analysis. Br J Anaesth 112(4):648-659

108. Srinivasa S, Taylor MH, Singh PP, Yu TC, Soop M, Hill AG (2013) Randomized clinical trial of goal-directed fluid therapy within an enhanced recovery protocol for elective colectomy. $\mathrm{Br} J$ Surg 100(1):66-74

109. Zheng H, Guo H, Ye JR et al (2013) Goal-directed fluid therapy in gastrointestinal surgery in older coronary heart disease patients: randomized trial. World J Surg 37(12):2820-2829

110. Grocott MP, Dushianthan A, Hamilton MA et al (2013) Optimisation systematic review steering group. Perioperative increase in global blood flow to explicit defined goals and outcomes after surgery: a Cochrane Systematic Review. Br JAnaesth 111(4):535-548

111. Pearse RM, Harrison DA, MacDonald N, et al, OPTIMISE Study Group (2014) Effect of a perioperative, cardiac output-guided hemodynamic therapy algorithm on outcomes following major gastrointestinal surgery: a randomized clinica trial and systematic review. Group (New York) 311(21):2181-2190

112. Wilms $H$, Mittal A, Haydock MD et al (2014) A systematic review of goal directed fluid therapy: rating of evidence for goals and monitoring methods. J Crit Care 29:204-209

113. Camacho Navarro LH, Bloomstone JA et al (2015) Perioperative fluid therapy: a statement from the international fluid optimization group. Perioper Med (Lond) 4:3

114. Lilot M, Ehrenfeld JM, Lee C et al (2015) Variability in practice and factors predictive of total crystalloid administration during abdominal surgery: retrospective two-centre analysis. $\mathrm{Br}$ J Anaesth 114(5):767-776

115. Schlembach D, Mörtl MG, Girard T, Arzt W, Brezinka C et al (2013) Management der postpartalen Blutung. Der „D-A-CH“-Algorithmus. Interdisziplinäre "D-A-CH"-Konsensusgruppe PPH (Deutschland Österreich - Schweiz). Schweiz Med Forum 13(50):1033-1038 
Hier steht eine Anzeige.

\section{曾 Springer}

